\title{
Renormalization of Proton-Proton Scattering and Proton-Proton Fusion with Chiral Two Pion Exchange: Regulator Independent Results.
}

\author{
M. Pavón Valderrama ${ }^{1, a}$ \\ Institut für Kernphysik and Jülich Center for Hadron Physics, Forschungszentrum Jülich, DE-52424 Jülich (Germany)
}

\begin{abstract}
The renormalization of the $s$-wave proton-proton scattering amplitude with chiral two-pion exchange is considered up to next-to-next-to-leading order in the chiral expansion. The resulting phase shifts accurately describe the $s$-wave phases up to $k_{c m} \simeq 200 \mathrm{MeV}$. The effect and possible improvements coming from finite cut-offs or spectral function regularization are considered. Proton-proton fusion is calculated with the resulting renormalized wave functions in a regulator independent way at next-to-next-to-leading order $\left(\mathrm{N}^{2} \mathrm{LO}\right)$.
\end{abstract}

\section{Introduction}

The application of effective field theory (EFT) techniques to the problem of nuclear forces provides the possibility of a systematic and model-independent formulation of nuclear physics which is consistent with the low energy symmetries of Quantum Chromodynamics (see [1,2] for recent reviews). The long range dominance of pion exchanges [3], which in turn are constrained by the requirements of broken chiral symmetry, suggests the feasibility of this approach. The seminal work by Weinberg $[4,5]$ made the first proposal for the implementation of a nuclear EFT: the idea is to compute the potential between nucleons according to the rules of chiral perturbation theory. This chiral potential in then iterated within the Schrödinger or the LippmannSchwinger equation in order to obtain theoretical predictions for observables. This approach was pioneered by the work of Ordóñez, Ray and van Kolck [6,7], triggering an intense activity which has eventually led to the development of chiral descriptions of the nuclear force $[8,9]$ which are able to compete on equal ground with the best phenomenological potentials.

However, the application of effective field theory techniques usually entails the appearance of singular interactions. Recent developments in the understanding of singular potentials [10-12] have shown that the Weinberg counting is not renormalizable, in the sense that it needs to be supplemented with higher order contact interactions in order to generate finite amplitudes when the regulator is removed. On the other hand, the fact that the cut-off dependence found in the original Weinberg counting is cured only by higher order operators suggests that it only corresponds to the natural short range uncertainty expected at a given order $[13,14]$. This has led to an interesting debate on whether one should modify $[10,15,16]$, or not $[13,14]$ the Weinberg counting to accommodate the previous observations. We see that in fact much of the recent discus-

\footnotetext{
a e-mail: m.pavon.valderrama@fz-juelich.de
}

sions on the formulation of EFT have been driven by an improved understanding of the special role which singular interactions have.

In this talk, I will consider the renormalization of the chiral potentials in the particular case of proton-proton (pp) scattering in the ${ }^{1} S_{0}$ singlet channel and proton-proton fusion. By renormalization I refer to (i) the possibility of expressing all observables in terms of other physical observables and the physical parameters of the long range interaction and (ii) achieving regulator independence over a reasonable range of cut-offs. Instead of the usual effective field theory formulation of the scattering problem in terms of long range potentials and counterterms, the fulfillment of the previous conditions will be realized on the base of the long range correlations [11] which chiral one and two pion exchange generate in the proton-proton system. In this approach, contact operators are treated implicitly, via boundary conditions in coordinate space, and will be given a secondary role in the discussion. Depending on the number of constraints imposed on the long range correlations, different power countings can be accommodated. The requirements of cut-off independence can also be easily analyzed within the long range correlation picture [11,12]: in the case of regular and singular attractive interactions, the dependence on the cut-off scale can be completely eliminated, but not so in the singular repulsive case, in which finite cut-offs are forcefully required. In this latter case, it is essential for the effective field theory description that the coordinate space cut-off at which the divergences appear is similar to or smaller than the short range scale $R_{S}$ of the system, i.e. $r_{c} \lesssim R_{S}$.

For fixing ideas, some examples of the kind of correlations I want to discuss in this talk are (i) the prediction of the $D / S$ asymptotic ratio of the deuteron, $\eta=0.02633$ (to be compared with $\eta_{\exp }=0.0256(4)$ ), from one pion exchange constraints and the binding energy of the deuteron [17], or (ii) the following correlation between the effective 
range $r_{0}$ and the scattering length $a_{0}$ for s-waves [11]

$$
r_{0}\left(a_{0}\right)=A+\frac{B}{a_{0}}+\frac{C}{a_{0}^{2}},
$$

which is fulfilled at a remarkable level of accuracy in the case of alkali atoms with van der Waals interaction, as has been recently discussed in Ref. [18]. This approach has also deep commonalities with the modified effective range expansion (see D. Minossi's talk in these proceedings) which will be discussed in a forthcoming publication [34].

\section{Renormalization of Chiral Two-Pion Exchange}

In the Weinberg counting the nucleon-nucleon (NN) potential is expressed as a low energy expansion which takes the form

$$
V_{\mathrm{NN}, \chi}(\mathbf{r})=V_{\chi}^{(0)}(\mathbf{r})+V_{\chi}^{(2)}(\mathbf{r})+V_{\chi}^{(3)}(\mathbf{r})+O\left(Q^{4}\right),
$$

where $Q$ generically represents the low energy scales of the system, for example the pion mass $m_{\pi}$ or the exchanged momentum $q$. Order $Q^{0}$ is usually referred to as leading order (LO), order $Q^{2}$ as next-to-leading order (NLO), order $Q^{3}$ as next-to-next-to-leading order $\left(\mathrm{N}^{2} \mathrm{LO}\right)$, etc.

The previous potential is composed of a finite and a contact range piece. The finite range piece consists of pion exchanges constrained by the requirements of chiral symmetry. The order $v$-th finite range contribution scales as $Q^{v}$, leading to a generic behaviour of $V^{(v)}(q) \sim|\mathbf{q}|^{v} f\left(q / m_{\pi}\right)$ in momentum space, with $f(x)$ a non-polynomial function ${ }^{1}$. The previous form generates coordinate space potentials which show power law divergences at short distances $\left(m_{\pi} r \ll 1\right)$, with $V^{(v)}$ behaving as $1 / r^{3+v}$. The contact range piece contributions appear only at even orders, $v=2 n$, and consist of delta potentials and their derivatives. Some sort of regularization is needed in order to make sense of the contact terms, and they are basically used as free parameters to fit the data. As we are interested here in the long range correlations generated by chiral pion exchanges, the role of the contact range interactions will be generally treated as secondary, and its counting will be violated in some cases.

\subsection{Renormalizing Singular Interactions}

The inverse power law behaviour of the chiral NN potentials is also in need of regularization. When treated perturbatively, it will give rise to divergences, as can be appreciated by direct evaluation of its matrix elements

$$
\left\langle\Psi^{(0)}\left|V_{\chi}^{(v)}\right| \Psi^{(0)}\right\rangle \sim \int_{r_{c}} d^{3} \mathbf{r} \frac{\left|\Psi^{(0)}(\mathbf{r})\right|^{2}}{r^{3+v}},
$$

1 The inclusion of static degrees of freedom, e.g. the $\Delta$ isobar in the small scale expansion, will slightly alter the previous behaviour. where $\left|\Psi^{(0)}\right\rangle$ represents the leading order s-wave wave function. The integral is divergent for any regular wave function, even for $v=0$. The treatment of these divergences involve making the necessary subtractions or including the relevant counterterms. Due to the the singular character of the perturbations and the distorted wave nature of the $\left|\Psi^{(0)}\right\rangle$ wave function, it has not been clear until recently (except for specific toy models [19]) how to construct such a renormalized perturbation theory for the particular case of chiral potentials [20]. In general perturbative treatments lead to an increased number of counterterms, a disadvantage which is compensated by the manifest power counting in the amplitudes (and not only in the potential) ${ }^{2}$, not to mention the compatibility of the counterterm structure with renormalization group analysis [15].

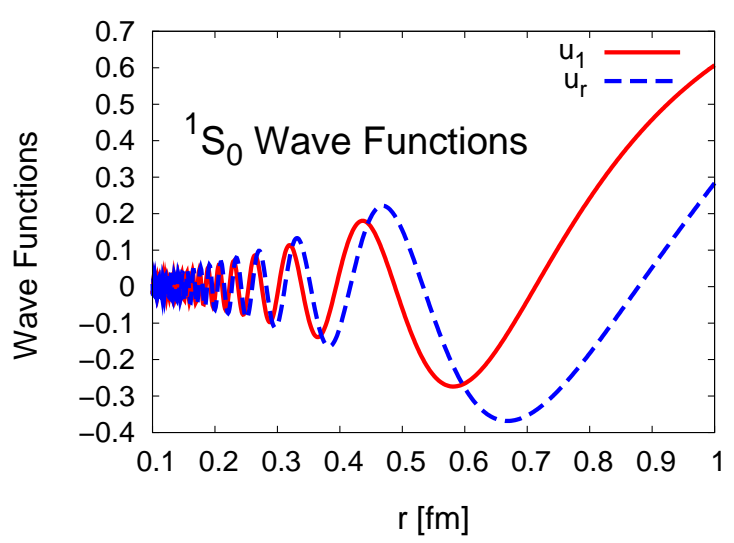

Fig. 1. Two linearly independent zero-energy solutions of the chiral TPE potential for the neutron-proton system. The $u_{1}\left(u_{r}\right)$ solution behaves asymptotically $(r \rightarrow \infty)$ as $\rightarrow 1(\rightarrow r)$. Near the origin, as $r \rightarrow 0$, both solutions behave regularly, approaching the limit $u(0)=0$.

On the contrary, when treated non-perturbatively, attractive singular potentials will generate ambiguities (instead of divergences). This can be better understood if we consider the reduced Schrödinger equation

$$
-u_{k}^{\prime \prime}+2 \mu\left[V(r)+\frac{l(l+1)}{r^{2}}\right] u_{k}(r)=k^{2} u_{k}(r),
$$

where $u_{k}$ is the reduced wave function, $V(r)$ the potential and $l$ the angular momentum. For regular potentials, we have two kinds of solutions at short distances $(r \rightarrow 0)$ : the regular solution, behaving as $u_{k, \text { reg }}(r) \sim r^{l+1}$, and the irregular one, $u_{k \text {,irr }}(r) \sim r^{-l}$. The physical solution is chosen to be the regular one, $u_{k}(0)=0$. But for an attractive singular potential, as the ones generated in chiral perturbation

\footnotetext{
2 This does not mean however that power counting is lost in non-perturbative computations, only that it is not evident. In that regard, it is interesting to notice the arguments of Ref. [13] about the systematicity of non-perturbative finite cut-off computations. Even if the regulator is removed, there is the possibility of using fractional scaling [11] as a means of making a priori error estimations.
} 


\section{$19^{\text {th }}$ International IUPAP Conference on Few-Body Problems in Physics}

theory, any solution of the Schrödinger equation is a regular solution near the origin, meaning that all of them are mathematically acceptable solutions. That can be appreciated in Fig. (1), where two different (linearly independent) solutions of the chiral two pion exchange (TPE) potential for neutron-proton scattering at $\mathrm{N}^{2} \mathrm{LO}$ are shown. Therefore the question is how to choose a physically meaningful wave function.

\subsection{Boundary Condition Renormalization}

The solution is to fix the ambiguities with some piece of physical information of the system. In effective theories we are interested in the low energy behaviour of a system. Then, the most natural choice is the s-wave scattering length. For simplicity I will assume a neutral system without Coulomb forces. In such a case it is straightforward to construct the zero energy solution of the system by integrating the Schrödinger equation from infinity to the cutoff radius, i.e.

$$
\begin{aligned}
-u_{0}^{\prime \prime}+2 \mu V(r) u_{0}(r) & =0, \\
u_{0}(r) & \rightarrow 1-\frac{r}{a_{0}} \quad \text { for } r \rightarrow \infty,
\end{aligned}
$$

where $a_{0}$ is the scattering length of the two-body system. For constructing the finite momentum solutions, I will assume an energy-independent boundary condition (BC) at the cut-off radius

$$
\frac{u_{k}^{\prime}\left(r_{c}\right)}{u_{k}\left(r_{c}\right)}=\frac{u_{0}^{\prime}\left(r_{c}\right)}{u_{0}\left(r_{c}\right)}+O\left(\frac{k^{2}}{\Lambda_{S}^{2}}\right),
$$

where the previous condition is supposed to be accurate up to order $k^{2} / \Lambda_{S}^{2}$, with $\Lambda_{S}$ is a high energy scale. Then we integrate upwards the Schrödinger equation from the cutoff radius to infinity in order to obtain the phase shifts,

$$
\begin{aligned}
-u_{k}^{\prime \prime}+2 \mu V(r) u_{k}(r) & =k^{2} u_{k}(r) \\
u_{k}(r) & \rightarrow F_{k} \frac{\sin (k r+\delta)}{\sin \delta} \quad \text { for } r \rightarrow \infty,(9)
\end{aligned}
$$

with $F_{k}$ some arbitrary normalization constant.

The boundary condition regularization (BCR) can be easily extended to higher partial waves or to more counterterms. Higher parameters of the effective range expansion, like $r_{0}, v_{2}, \ldots v_{n}$, can be fixed by integrating the corresponding Schrödinger equations for $k^{2}, \ldots, k^{2 n}$ from infinity to the cut-off radius. The resulting boundary condition is

$$
\begin{aligned}
\frac{u_{k}^{\prime}\left(r_{c}\right)}{u_{k}\left(r_{c}\right)} & =\frac{u_{0}^{\prime}\left(r_{c}\right)+k^{2} u_{2}^{\prime}\left(r_{c}\right)+\ldots+k^{2 n} u_{2 n}^{\prime}\left(r_{c}\right)}{u_{0}\left(r_{c}\right)+k^{2} u_{2}\left(r_{c}\right)+\ldots+k^{2 n} u_{2 n}\left(r_{c}\right)} \\
& +O\left(\frac{k^{2 n+2}}{\Lambda_{S}^{2 n+2}}\right) .
\end{aligned}
$$

This boundary condition has the unique property of being the only one generating finite results for attractive singular interactions when the cut-off is removed $\left(r_{c} \rightarrow 0\right)$ [23]. No other short distance boundary condition has this property if attractive singular potentials are present. Unfortunately, the equivalent representation for the Lippmann-Schwinger equation has not been found yet and the usual representation of the short distance potential, $C_{0}+C_{2}\left(p^{2}+p^{\prime 2}\right)$, does not yield well-defined results in the $\Lambda \rightarrow \infty$ limit [24]. The relationship between the energy derivative of the boundary condition and the Wigner bound is discussed in Ref. [11].

A trivial example of the previous procedure is given by pionless theory, in which we set $V(r)=0$ for $r>r_{c}$. In such a case, boundary condition regularization yields the following solution when one parameter is fixed

$$
k \cot \left(\delta+k r_{c}\right)=\frac{1}{r_{c}-a_{0}} .
$$

This expression reproduces the first term of the effective range expansion, $k \cot \delta=-1 / a_{0}+O\left(k^{2} r_{c}\right)$. It is straightforward to fix more parameters, obtaining, for small $r_{c}$, the following

$$
\begin{aligned}
k \cot \delta & =-\frac{1}{a_{0}}+\frac{1}{2} r_{0} k^{2}+\ldots+v_{2 n} k^{2 n} \\
& +O\left(k^{2 n+2} R_{S}^{2 n} r_{c}\right)
\end{aligned}
$$

where $R_{S}$ is the typical scale corresponding to the effective range expansion parameters, which in the present example is treated as a short range scale. As far as $r_{c} \lesssim R_{S}$, the previous expression will generate error estimations which are consistent with power counting.

\subsection{Boundary Conditions as a Representation of Short Range Physics}

Boundary conditions provide a general representation of short range physics for non-relativistic scattering problems. The reason for that is that the reduced Schrödinger equation is a second order differential equation: therefore the value of the wave function and its derivative at some radius $r_{c}$ completely determines the solution. Whatever the physics of the problem is for $r<r_{c}$, a boundary condition at $r=r_{c}$ will encode it. Boundary conditions are then basically equivalent to counterterms, and have been used in many effective field theory works. They are not constrained to computations where small cut-offs $\left(m_{\pi} r_{c} \ll 1\right)$ are used. In fact, boundary conditions naturally fit into the renormalization philosophy of Lepage [21] as a way of parametrizing unknown short range effects. A recent example of this is provided by Ref. [22], where the scaling of the logarithmic boundary condition is used to determine which short range physics to include when chiral TPE is treated perturbatively in the $1.0<r_{c}<1.8 \mathrm{fm}$ range.

\subsection{Why it is Renormalization and not only Regularization}

The previous boundary condition method does not only regularize the amplitudes, it also renormalizes them, as it 
enable us to express all the physical amplitudes in terms of low energy observables and functions which depend solely on the long range physics. In other words, boundary condition regularization is a Wilsonian renormalization approach which describes the long range distortion of short range physics

$$
\delta_{S}(k) \rightarrow \text { (long range distortion) } \rightarrow \delta(k),
$$

and where the cut-off $r_{c}$ plays the role of the separation scale between long and short range effects. In such a case, the renormalization group equation for the boundary condition when the requirement of reproducing the physical phase shifts $\delta(k)$ is added, is the following one [23]

$$
L_{k}^{\prime}\left(r_{c}\right)+L_{k}^{2}\left(r_{c}\right)=2 \mu V\left(r_{c}\right)-k^{2},
$$

with $L_{k}\left(r_{c}\right)=u_{k}^{\prime}\left(r_{c}\right) / u_{k}\left(r_{c}\right)$ the logarithmic boundary condition. The short range phase shift can then be extracted from $L_{k}\left(r_{c}\right)=k \cot \left(k r_{c}+\delta_{S}\right)$. Of course, if we are fixing the first $n$ terms of the effective range expansion, only the first $n$ terms of the $k^{2}$ expansion of the previous renormalization group equation will be preserved, guaranteeing renormalization group invariance up to order $k^{2 n+2}$.

An interesting application of the boundary condition RGA of Ref. [23] is that one can determine the cut-off dependence of observables when they are renormalized with $n$ fixed parameters, Eq. (10), yielding

$$
\lim _{r_{c} \rightarrow 0} \frac{d \delta\left(k ; r_{c}\right)}{d r_{c}}=-k^{2 n+1} \sum_{j=0}^{2 n} u_{2 j}\left(r_{c}\right) u_{2 j-2 n}\left(r_{c}\right) .
$$

For attractive singular potentials, behaving as $-1 / r^{N}$, the previous formula predicts a convergence rate of $\Delta \delta\left(r_{c}\right) \sim$ $r_{c}^{N / 2+1}$, a trend which is independent of the number of fixed parameters.

\subsection{Long Range Correlations}

In combination with the superposition principle, boundary condition regularization gives rise to long range correlation between observables. To illustrate this point we can consider the simplest boundary condition possible

$$
\frac{u_{k}^{\prime}\left(r_{c}\right)}{u_{k}\left(r_{c}\right)}=\frac{u_{0}^{\prime}\left(r_{c}\right)}{u_{0}\left(r_{c}\right)},
$$

where $u_{0}$ and $u_{k}$ are solutions of Eqs. (5) and (8). By using the superposition principle, the zero energy wave function can be written as follows

$$
u_{0}(r)=u_{1}(r)-\frac{u_{r}(r)}{a_{0}},
$$

where $u_{1}$ and $u_{r}$ are zero energy solutions subjected to the asymptotic $(r \rightarrow \infty)$ boundary conditions $u_{1} \rightarrow 1$ and $u_{r} \rightarrow r$. Conversely, for the finite energy wave function we can write

$$
u_{k}(r)=u_{c}(r)+k \cot \delta u_{s}(r)
$$

where $u_{c}$ and $u_{s}$ behave asymptotically as $u_{c} \rightarrow \cos (k r)$ and $u_{s} \rightarrow \sin (k r) / k$. Inserting the previous equations, Eqs. (17) and (18), into the boundary condition Eq. (7), one gets

$$
k \cot \delta=\frac{-\mathcal{A}\left(k ; r_{c}\right) / a_{0}+\mathcal{B}\left(k ; r_{c}\right)}{-C\left(k ; r_{c}\right) / a_{0}+\mathcal{D}\left(k ; r_{c}\right)},
$$

where $\mathcal{A}\left(k ; r_{c}\right), \mathcal{B}\left(k ; r_{c}\right), \mathcal{C}\left(k ; r_{c}\right)$ and $\mathcal{D}\left(k ; r_{c}\right)$ are functions which depend solely on the long range potential $V(r)$, the momentum $k$ and the cut-off $r_{c}$, and which can be expressed as Wronskian relationships between the zero and finite energy wave functions

$$
\begin{aligned}
& \mathcal{A}\left(k ; r_{c}\right)=-\left.W\left(u_{r}, u_{c}\right)\right|_{r=r_{c}}, \\
& \mathcal{B}\left(k ; r_{c}\right)=-\left.W\left(u_{1}, u_{c}\right)\right|_{r=r_{c}}, \\
& \mathcal{C}\left(k ; r_{c}\right)=\left.W\left(u_{r}, u_{s}\right)\right|_{r=r_{c}}, \\
& \mathcal{D}\left(k ; r_{c}\right)=\left.W\left(u_{1}, u_{s}\right)\right|_{r=r_{c}},
\end{aligned}
$$

where $\left.W(f, g)\right|_{r=r_{c}}=f\left(r_{c}\right) g^{\prime}\left(r_{c}\right)-f^{\prime}\left(r_{c}\right) g\left(r_{c}\right)$. This correlation was originally described in Ref. [11] and can be easily extended to include additional counterterms just following the steps presented above to the more general case in which the first $n$ parameters of the effective range expansion are fixed, see Ref. [25].

\section{Phase Shifts}

The proton-proton system is described in the singlet channel with the following $s$-wave reduced Schrödinger equation

$$
-u_{k}^{C^{\prime \prime}}(r)+2 \mu\left[V_{N}+V_{C}(r)\right] u_{k}^{C}(r)=k^{2} u_{k}^{C}(r),
$$

where $\mu=M_{p} / 2$ is the reduced mass, $k$ the center-of-mass (c.m.) momentum, $V_{N}$ the strong pp potential and $V_{C}=\frac{\alpha}{r}$ the Coulomb potential ${ }^{3}$, with $\alpha$ the fine structure constant. The above equation is assumed to be valid for $r>r_{c}$, while at $r=r_{c}$ the short range physics is parametrized by a boundary condition. The pp Coulomb phase shifts are determined from the asymptotic $(r \rightarrow \infty)$ behaviour of the wave function $u_{k}^{C}$

$$
u_{k}^{C}(r) \rightarrow \cot \delta^{C} F_{0}(\eta, \rho)+G_{0}(\eta, \rho),
$$

with $\delta^{C}$ the Coulomb phase shift, and $F_{0}$ and $G_{0}$ the regular and irregular Coulomb wave functions, which are the solutions of Eq. (24) with $V_{N}=0$. They depend on the parameters $\eta=M_{p} \alpha / k$ and $\rho=k r$.

The low energy behaviour of pp scattering is described by the Coulomb effective range expansion, defined by

$$
\begin{aligned}
C^{2}(\eta) k \cot \delta_{0}^{C}+2 k \eta h(\eta) & =-\frac{1}{a_{0}^{C}}+\frac{1}{2} r_{0}^{C} k^{2} \\
& +\sum_{n=2}^{\infty} v_{n}^{C} k^{2 n},
\end{aligned}
$$

3 The inclusion of corrections to the electromagnetic interaction between nucleons, like for example improved Coulomb [26] or vacuum polarization [27], is not needed at the present level of accuracy. As can be seen in Ref. [28], the previous effects generate at most a correction of half a degree in the phase shifts at low energies. 
with $a_{0}^{C}$ the Coulomb scattering length, $r_{0}^{C}$ the effective range, $v_{2}^{C}$ the shape parameter, etc. The functions $C^{2}(\eta)$ and $h(\eta)$ are given by

$$
\begin{aligned}
C^{2}(\eta) & =\frac{2 \pi \eta}{e^{2 \pi \eta}-1}, \\
h(\eta) & =\eta^{2} \sum_{n=1}^{\infty} \frac{1}{n\left(n^{2}+\eta^{2}\right)}-\log \eta-\gamma_{E},
\end{aligned}
$$

with $\gamma_{E}$ the Euler-Mascheroni constant. The Coulomb effective range expansion converges for $k<m_{\pi^{0}} / 2$, with $m_{\pi^{0}}$ the neutral pion mass.

The renormalization is done essentially in the way described in Sect.(2), although care must be taken in changing all the free wave functions by their Coulomb counterparts. Here I generate results with one and two effective range parameters fixed, namely the scattering length and the effective range $\left(a_{0}^{C}=-7.815 \mathrm{fm}\right.$ and $r_{0}^{C}=2.76 \mathrm{fm}$ for the Nijmegen II potential [29]). The exact form of the chiral $\mathrm{N}^{2}$ LO pp potential is taken from Ref. [3], and the following values are used in the numerical computations: $f_{\pi}=92.4 \mathrm{MeV}, m_{\pi^{0}}=134.98 \mathrm{MeV}, M_{p}=938.27 \mathrm{MeV}$, $g_{A}=1.29$ in the LO piece of the potential (due to the Goldberger-Treiman discrepancy) and $g_{A}=1.26$ in the subleading pieces. For the chiral couplings we use the socalled "set II" in the notation of Ref. [11] $\left(c_{1}=-0.76 \mathrm{GeV}^{-1}\right.$, $c_{3}=-5.08 \mathrm{GeV}^{-1}, c_{4}=4.70 \mathrm{GeV}^{-1}$ ), which corresponds the determination of Rentmeester, Timmermans and de Swart [41].

The results are shown in Fig. (2). In Panel (a) only the Coulomb scattering length is fixed, while in Panel (b) the effective range is also fixed for the NLO and $\mathrm{N}^{2} \mathrm{LO}$ results (corresponding to the standard Weinberg counting for the short range operators). It can be appreciated that a convergence pattern arises, which unfortunately clearly underpredicts the phase shifts at higher orders. In principle it would be plausible to think that the $\mathrm{N}^{3} \mathrm{LO}$ contributions will solve the previous problem, but recent computations in the neutron-proton case seems to indicate the contrary [24]. In the following section I will try to look for the missing physics which generate this discrepancy. Apart from that, it is quite interesting to notice that there is little difference between fixing one or two parameters. This is explained by the fact that one parameter alone is enough to renormalize the phase shifts, indicating that the relevant short range physics have been already included with that single counterterm. Therefore it is not surprising that the second counterterm has such a small effect.

In fact, the contribution of the second counterterm can be numerically assessed by noticing that in a boundary condition context the (Coulomb) effective range $r_{0}^{C}$ can be expressed as a sum of long and short range effects

$$
r_{0}^{C}=r_{0, L}^{C}+r_{0, S}^{C},
$$

where $r_{0, L}^{C}\left(r_{0, S}^{C}\right)$ represent the long (short) range contribution to the effective range. The long range piece obeys the Coulomb counterpart of Eq.(1), that is

$$
r_{0, L}^{C}\left(a_{0}^{C}\right)=A+\frac{B}{a_{0}^{C}}+\frac{C}{a_{0}^{C^{2}}},
$$
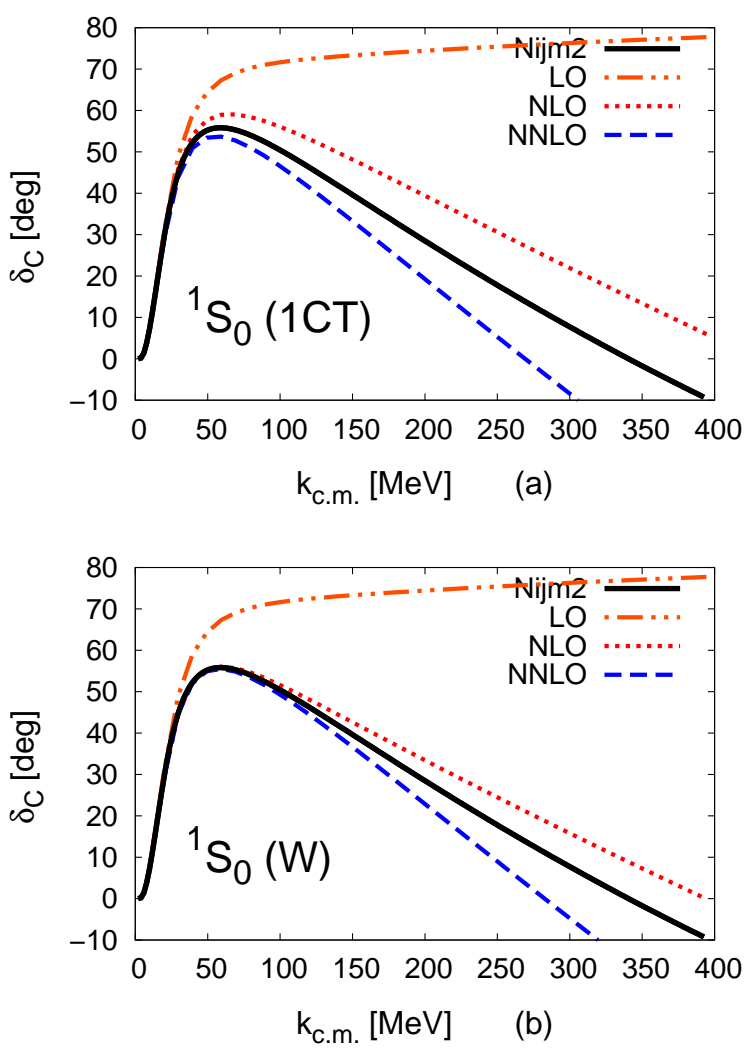

Fig. 2. The ${ }^{1} S_{0}$ Coulomb pp phase shifts with the chiral potentials from LO to $\mathrm{N}^{2} \mathrm{LO}$ when the cut-off radius is removed $\left(r_{c}=0\right)$. Panel (a) shows the phase shifts when only the Coulomb scattering length is fixed $\left(a_{0}^{C}=-7.815 \mathrm{fm}\right)$ and Panel (b) shows the result when the counterterms are included according to the Weinberg counting, resulting in two counterterms (or renormalization conditions) for the NLO and $\mathrm{N}^{2} \mathrm{LO}$ computations. The second condition is used to fix the Coulomb effective range to the $\mathrm{Ni}$ jmegen II value $\left(r_{0, \text { ijm }}^{C}=2.76 \mathrm{fm}\right)$.

while the short range piece is essentially unconstrained and can have any value. The relative contribution of the second counterterm to the effective range is therefore $\left(r_{0}^{C}-r_{0, L}^{C}\right) / r_{0}^{C}$, roughly giving a $13 \%$ contribution at NLO and a $-5 \%$ at $\mathrm{N}^{2} \mathrm{LO}$.

\section{The Missing Scales}

In this section I try to determine what is missing from the $\mathrm{N}^{2} \mathrm{LO}$ computation if one wants to reproduce the phase shifts, although the right question, as we will see, is not what is missing, but rather of what there is too much.

\subsection{Finite Cut-off Computations}

As is well known, the effective field theory description of nuclear forces has achieved at $\mathrm{N}^{3} \mathrm{LO}[8,9]$ levels of precision comparable to those of the so-called high precision potentials. Even most $\mathrm{N}^{2} \mathrm{LO}$ computations $[32,33]$ achieve 
(at least for the neutron-proton scattering case) an accuracy better than that shown in Fig. (2) and the main property of these computations is that they use a finite cut-off. In fact it has been argued that a finite cut-off should be a fundamental ingredient for a consistent formulation of the Weinberg counting $[13,14]$. Therefore I will explore this idea.

There are multiple ways to implement a finite cut-off. An advantage of knowing the $r_{c} \rightarrow 0$ limit is that it allows for a much more complete analysis of the cut-off dependence. Only those regulators having a well-defined $r_{c} \rightarrow 0$ limit will be considered, The finite cut-off regulators can be roughly divided in two categories: those which converge from below the renormalized results and those which converge from above. An example of a regulator which converges from above is the potential regulator

$$
V\left(r ; r_{c}\right)=V(r)\left(1-e^{-\left(r / r_{c}\right)^{6}}\right) .
$$

This regulator is then combined with a boundary condition at the origin and has the property of having a well-defined limit for $r_{c} \rightarrow 0^{4}$. For a regulator converging from below, a straightforward example is already provided by the boundary condition method described in Sect.(2).

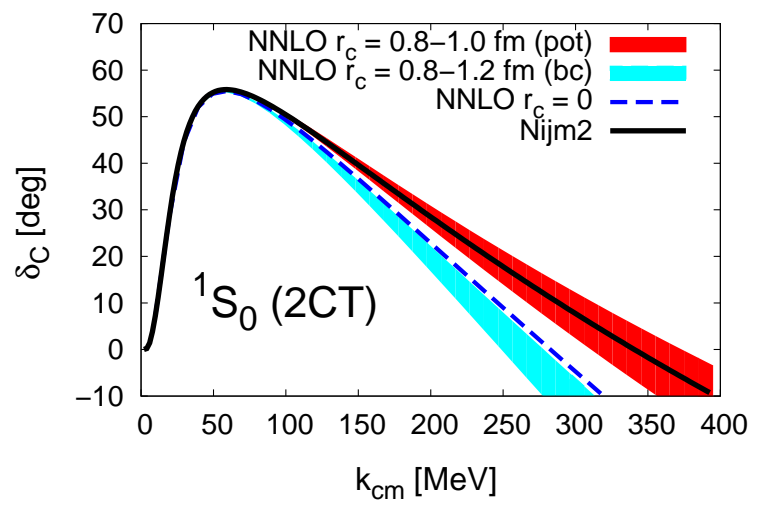

Fig. 3. Cut-off dependence of the ${ }^{1} S_{0}$ Coulomb pp phase shifts for two different regulators, one converging from below and the other from above the $r_{c}=0$ result. The calculations are done at $\mathrm{N}^{2} \mathrm{LO}$ with the Coulomb scattering length and effective range fixed to the Nijmegen II values. The red band uses the potential regulator described by Eq. (31), while the light blue band uses a boundary 1 condition at $r=r_{c}$.

The results of applying these two regulators can be seen in Fig. (3). As expected, the regulator converging from above reproduces the Nijmegen II phase shifts for cut-offs in the range $0.8-1.0 \mathrm{fm}$. For the boundary condition regulator, larger cut-offs need to be used in order to see any significant deviation with respect to the $r_{c}=0$ calculation, and the results are then slightly worse. The best possible cut-off for this regulator is $r_{c}=0$. As both regulators are equally good on a theoretical ground, one is bound to

\footnotetext{
${ }^{4}$ This property should be explicitly checked, as other regulators do not have it, for example $V\left(r ; r_{c}\right)=V(r)\left(1-e^{-\left(r / r_{c}\right)^{4}}\right)$.
}

ask: which should be the correct error estimation associated with finite cut-off calculations? The answer seems to be at least the difference between the $r_{c}=0$ phase shifts and the experimental ones.

The reason why only one of the regulators gives a satisfactory answer for finite cut-offs seems to be that the potential is excessively attractive. In that regard, I will consider the use of the spectral function regularization in the next section, representing a different example of a regulator which weakens the chiral two pion exchange potential.

Of course, this is a problem for the effective theory formulation of nuclear forces. An important detail which should be taken into account here is the length scale involved in the convergence to the $r_{c} \rightarrow 0$ limit. As can be appreciated from Fig. (3), this scale is of the order of $\sim 1 \mathrm{fm}$, which is a long distance scale. Would have it converged with respect to a different scale, say $\sim 0.25 \mathrm{fm}$ (about $\left.\sim 1 / m_{\rho}\right)$, and things would have been different. Consequently on this particular case there seems to be no stringent reason why one should distrust the results coming from the regulator which does not reproduce the data. In fact, there is no reason either why finite cut-offs should be preferred. If regulator dependence and renormalizability are taken into account together, one can always construct a regulator which good convergence properties and which always yields worse results than the $r_{c}=0$ limit for moderate cut-offs $m_{\pi} r_{c} \sim 1$.

Therefore, finite cut-offs are only a satisfactory solution if one can find a compelling reason for using certain types of regulators. In this respect, an interesting possibility which will be explored in future works [34] is provided by the use of modified effective range expansion techniques together with the renormalization group ideas of Barford and Birse [35].

\subsection{Spectral Function Regularization}

Another possibility is to study the effect of the spectral function regularization (SFR), which was introduced in Ref. [36] as a tool to improve the convergence of nuclear effective theory, and which has been used in many recent works [31,37-39] with good results in general. The spectral function regularization is based on including a cut-off in the spectral function representation of the potential (based on the dispersion relations for $N \bar{N} \rightarrow \pi \pi$ ). For example, for the central piece of the chiral TPE potential, the spectral function representation is given by

$$
V_{C}(r)=\frac{1}{2 \pi^{2} r} \int_{2 m}^{\infty} d \mu \mu \rho_{C}(\mu),
$$

plus the corresponding representations for the spin-spin, tensor and spin-orbit pieces, and where $\rho_{C}(\mu)=\operatorname{Im} \hat{V}_{C}(i \mu)$ is the spectral function (SF), with $\hat{V}_{C}(|\mathbf{q}|)$ the momentum space version of the potential. Then, the SF regularized potential results simply from cutting the SF representation, i.e.

$$
V_{C}(r, \tilde{\Lambda}) \rightarrow \frac{1}{2 \pi^{2} r} \int_{2 m}^{\tilde{\Lambda}} d \mu \mu \rho_{C}(\mu)
$$




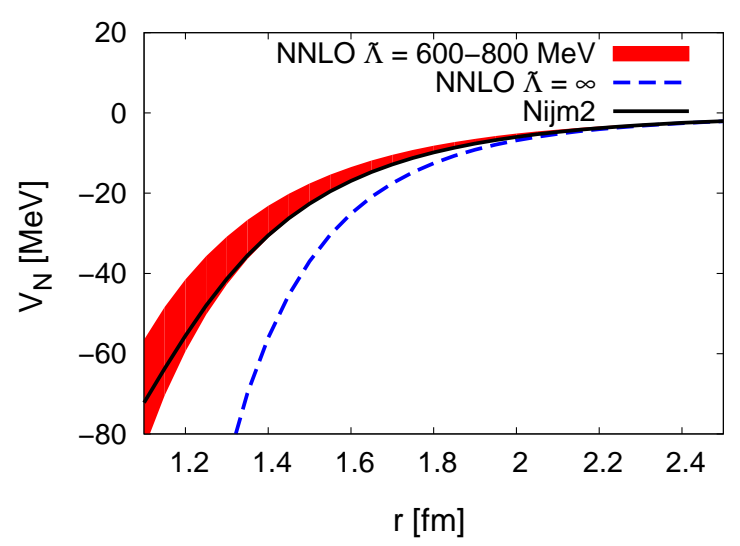

Fig. 4. The chiral TPE potential in dimensional regularization (dashed blue line), compared with the spectral function regularized one (red band) and with the Nijmegen II potential [29] (solid line). The band corresponds to changing the spectral cut-off in the range $\tilde{\Lambda}=600-800 \mathrm{MeV}$.

with $\tilde{\Lambda}$ the spectral cut-off.

The main effect of the spectral function regularization is to weaken the potential at short distances. In that sense, it is in principle an idea compatible with the effective field theory formulation of nuclear forces, although the distortion of the potential is sometimes too long ranged. This can be seen in Fig. (4), where the spectral function regularized potential is plotted in comparison with the dimensionally regularized one. It can be appreciated that SFR generates a sizeable distortion even for distances of the order of $\sim 1.5-2.0 \mathrm{fm}$, which are comparable to the pion Compton wavelength, $\sim 1.4 \mathrm{fm}$.

In Fig. (5) the phase shifts obtained with SFR are plotted for the $\mathrm{N}^{2} \mathrm{LO}$ potential with two renormalization conditions. A spectral cut-off between $600-800 \mathrm{MeV}$ is employed and the boundary condition cut-off is removed. As can be seen, the Nijmegen II phase shifts are reproduced within the error band. The fact that the results are so similar as with the potential regulator given in Eq. (31) suggests that the strong distortion of the potential at distances of the order of $m_{\pi} r \sim 1$ is not worrisome. The corresponding potentials shown in Fig. (4), indicate that the spectrally regularized potential behaves much more like the Nijmegen II potential [29] at large distances, explaining the improvement of the results over the dimensionally regularized potential. Therefore we see that the key to reproducing the phase shift is the weakening of the chiral TPE potential in this channel, as happened with the finite cut-off case.

\section{Proton-Proton Fusion}

The proton-proton fusion cross section is given by the following expression (see, for example, Ref. [40])

$$
\sigma\left(p p \rightarrow d v_{e} e^{+}\right)=\frac{1}{2 \pi^{3}} \frac{G_{V}^{2}}{v_{p p}} m_{e}^{5} f(E) \frac{1}{4} \sum_{m_{d}}\left|\left\langle d, m_{d}\left|\mathbf{A}_{-}\right| p p\right\rangle\right|^{2},
$$

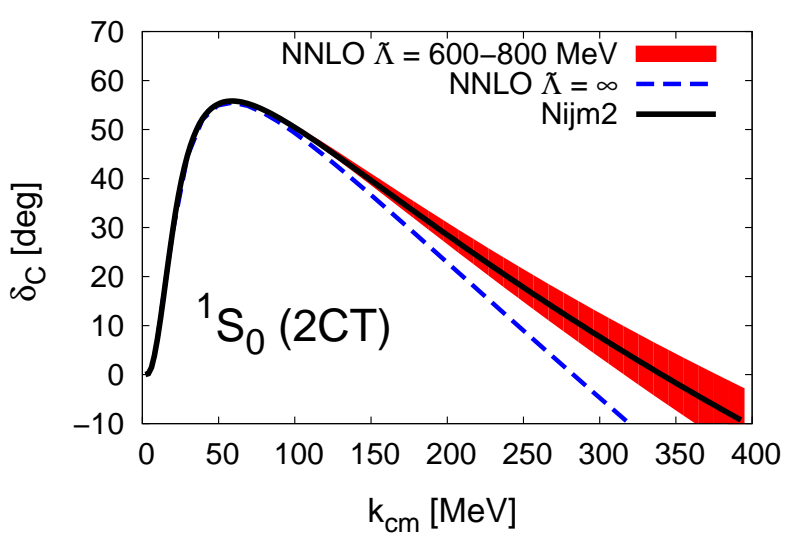

Fig. 5. Coulomb phase shifts for ${ }^{1} S_{0}$ pp scattering with spectral function regularization. The counterterms are fitted to reproduce the Coulomb scattering length and effective range and the band corresponds to varying the spectral cut-off in the $\tilde{\Lambda}=$ $600-800 \mathrm{MeV}$ window.

where $v_{p p}$ is the relative velocity of the protons in the center of mass frame, $m_{e}$ the electron mass, $f(E)$ the Fermi function, $m_{d}$ the third component of the deuteron spin and $G_{V}=1.14939 \cdot 10^{-5} \mathrm{GeV}^{-2}$. The axial operator $\mathbf{A}_{-}$is given in the impulse approximation by $\mathbf{A}_{-}^{1 B}=-g_{A} \sum_{i} \sigma_{i}\left(\tau_{1}-\right.$ $\left.\tau_{2}\right)_{i} / 2$, with $g_{A}=1.2654$ and where the index $i$ runs over the two nucleons. The corresponding matrix elements for the $\mathbf{A}_{-}^{1 B}$ operator can be written as

$$
\left\langle d, m_{d}\left|\mathbf{A}_{-}^{1 B}\right| p p\right\rangle=\left(\hat{r}_{\mu=m_{d}}\right)^{\dagger} g_{A} \sqrt{\frac{32 \pi}{\gamma^{3}}} C(\eta) \Lambda(E),
$$

with $\gamma=0.231605 \mathrm{fm}^{-1}$ the deuteron wave number, $\hat{r}_{\mu}$ the unit vectors in the spherical base and $\Lambda(E)$ the overlap integral, defined as

$$
\Lambda(E)=\sqrt{\frac{a_{0}^{C^{2} \gamma^{3}}}{2}} \int_{0}^{\infty} d r u_{d}(r) u_{p p, k}^{C}(r),
$$

where the asymptotic normalizations of the deuteron and proton-proton wave functions have been taken as $u_{d}(r) \rightarrow$ $A_{S} e^{-\gamma r}\left(A_{S}\right.$ is the s-wave asymptotic normalization of the deuteron) and $u_{p p . k}^{C}(r) \rightarrow C(\eta)\left(\cot \delta^{C} F_{0}(\eta, \rho)+G_{0}(\eta, \rho)\right)$. In general we are interested in the $E=0$ value of the overlap integral.

The $\mathrm{N}^{2} \mathrm{LO}$ deuteron wave functions are those from Ref. [11], and the resulting deuteron properties are shown in Table (1). These wave functions are constructed to reproduce the binding energy and the $D / S$-asymptotic ratio $\eta$ of the deuteron. We consider two different determinations of chiral coefficients, namely "set II", which has been used in the previous sections for computing the pp phase shifts, corresponding to the determination of Ref. [41], and "set IV" $\left(c_{1}=-0.81 \mathrm{GeV}^{-1}, c_{3}=-3.20 \mathrm{GeV}^{-1}, c_{4}=5.40 \mathrm{GeV}^{-1}\right)$, which was used in the $\mathrm{N}^{3} \mathrm{LO}$ calculation by Entem and Machleidt [8]. For comparison purposes we include also the LO wave functions of Ref. [17]. The pp wave functions are constructed to reproduce the pp Coulomb scat- 
Table 1. Deuteron properties for the LO and $\mathrm{N}^{2} \mathrm{LO}$ chiral potentials. The counterterms are fitted to reproduce the binding energy $\left(\mathrm{LO} / \mathrm{N}^{2} \mathrm{LO}\right)$ and the $\mathrm{D} / \mathrm{S}$ asymptotic ratio $\left(\mathrm{N}^{2} \mathrm{LO}\right)$. The experimental values for the observables are $\gamma=0.231605 \mathrm{fm}^{-1}$, $\eta=0.0256(4), A_{S}=0.8838(4) \mathrm{fm}^{-1 / 2}, r_{m}=1.971(5) \mathrm{fm}$, $Q_{d}=0.2860(15) \mathrm{fm}^{2}$ (see Ref. [31] and references within).

\begin{tabular}{lccc}
\hline & $\mathrm{LO}$ & $\mathrm{N}^{2} \mathrm{LO}($ set II) & $\mathrm{N}^{2} \mathrm{LO}($ set IV) \\
\hline$\gamma\left(\mathrm{fm}^{-1}\right)$ & Input & Input & Input \\
$\eta$ & 0.02633 & Input & Input \\
$A_{S}\left(\mathrm{fm}^{-1 / 2}\right)$ & $0.8681(1)$ & $0.900(2)$ & $0.884(4)$ \\
$r_{m}(\mathrm{fm})$ & & & \\
$\quad 1 \mathrm{~B}$ & $1.9343(5)$ & $1.999(4)$ & $1.967(6)$ \\
$\quad+2 \mathrm{~B}$ & $1.9406(3)$ & $2.004(4)$ & $1.972(7)$ \\
$Q_{d}\left(\mathrm{fm}^{2}\right)$ & & & \\
$1 \mathrm{~B}$ & $0.2762(1)$ & $0.284(5)$ & $0.276(3)$ \\
$\quad+2 \mathrm{~B}$ & $0.2878(1)$ & $0.294(4)$ & $0.286(3)$ \\
$P_{D}(\%)$ & $7.31(1)$ & $7(1)$ & $8(1)$ \\
\hline
\end{tabular}

tering length as explained in previous sections. In the following computations the cut-off has been eliminated. Although this lowers the range of applicability of the description in singlet pp scattering to $k<200 \mathrm{MeV}$, I am considering here a process at $k=0$, and consequently taking $r_{c} \rightarrow 0$ poses no problem for the validity of the effective description of pp fusion.

For these wave functions direct evaluation of the previous expression yields the value $|\Lambda(0)|^{2}=7.03(5)$ for set II of chiral couplings, and $|\Lambda(0)|^{2}=6.87(6)$ for set IV, where the error comes from the uncertainty in the $\mathrm{D} / \mathrm{S}$-asymptotic ratio $\eta=0.0256(4)$. As can be appreciated these values vary considerably depending on the chiral couplings considered, but the value of set II is more similar to the usual values obtained with phenomenological wave functions in the impulse approximation (ignoring any electromagnetic effect beyond Coulomb), which is 6.985 for the CD-Bonn potential and 6.965 for AV18 [42].

Of course the previous value is incomplete, as it only represents the contribution from the impulse approximation. A complete computations needs to include additional effects like electromagnetic corrections or two body currents. It is customary to express the contribution of any given effect $x$ as

$$
\delta_{x}=\frac{\Lambda_{x}-\Lambda}{\Lambda},
$$

where $\Lambda$ is the basic overlap integral of Eq. (36). The total value of the squared overlap integral is simply $\tilde{\Lambda}^{2}=$ $\Lambda^{2}(1+\delta)^{2}$, with $\delta$ the sum of all the $\delta_{x}$ 's coming from different contributions. The relative contribution from the different effects considered in this talk are summarized in Table (2).

The inclusion of improved Coulomb [26] or vacuum polarization effects [27] produces only small changes in the overlap integral, namely, $\delta_{v p}=-0.44 \%$ and $\delta_{C 2}=$ $0.03 \%$ at $E=0$. These values are quite similar to those usually obtained in the literature $[42,43]$.

The two body $O\left(Q^{3}\right)$ chiral contributions [44] to pp fusion can be divided in a finite range and contact range con-
Table 2. The squared proton-proton fusion overlap integral at zero energy, $|\Lambda(0)|^{2}$ for chiral wave functions computed at different orders, and the contributions $\delta_{x}=\left(\Lambda_{x}-\Lambda\right) / \Lambda$ coming from different effects. The contribution from vacuum polarization, improved Coulomb and the finite range piece of the chiral $O\left(Q^{3}\right)$ current are considered. The results are shown for the LO and $\mathrm{N}^{2} \mathrm{LO} \mathrm{pp}$ and deuteron wave functions The input parameters of the computation are the pp Coulomb scattering length, $a_{p p}^{C}=-7.815 \mathrm{fm}$, the deuteron wave number $\gamma=0.231605 \mathrm{fm}^{-1}$ and $\mathrm{D} / \mathrm{S}$ asymptotic ratio $\eta=0.0256(4)$. In the LO case, the error is estimated by varying the cut-off in the $r_{c}=0.1-0.2 \mathrm{fm}$ window. For the $\mathrm{LO}$ wave functions, the error comes from propagating the uncertainty in $\eta$. The value $|\tilde{\Lambda}(0)|^{2}$ contains only those contributions which can be computed in each case.

\begin{tabular}{lccc}
\hline & $\mathrm{LO}$ & $\mathrm{N}^{2} \mathrm{LO}($ set II) & $\mathrm{N}^{2} \mathrm{LO}($ set IV) \\
\hline$|\Lambda(0)|^{2}$ & $7.06(1)$ & $7.03(5)$ & $6.87(6)$ \\
$\delta_{\mathrm{vp}}(\%)$ & -0.58 & -0.44 & -0.44 \\
$\delta_{\mathrm{C} 2}(\%)$ & 0.10 & 0.03 & 0.03 \\
$\delta_{2 \mathrm{~B}(\mathrm{~F})}(\%)$ & - & $3.3(2)$ & $3.7(3)$ \\
$|\tilde{\Lambda}(0)|^{2}$ & $6.98(1)$ & $7.43(2)$ & $7.33(3)$ \\
\hline
\end{tabular}

tribution. The inclusion of the finite range two body operators is straightforward and yields finite results for $r_{c} \rightarrow 0$ in the case of the $\mathrm{N}^{2} \mathrm{LO}$ deuteron and pp wave functions. We obtain $\delta_{2 B} \simeq 3.3(2) \%$, which is much larger than the usual value with phenomenological currents and phenomenological (AV18) wave functions [42], $\delta_{2 B} \simeq 0.7 \%$, but smaller than the value obtained when evaluating chiral currents with the AV18 wave functions and removing the coordinate space cut-off [43], yielding $\delta_{2 B} \simeq 5.2 \%$. Nowadays it is more common to keep a finite cut-off in hybrid computations in which case a $\delta_{2 B} \simeq 1.6-2.7 \%$ is obtained for the AV18 wave functions in the momentum cut-off range $\Lambda=600-800 \mathrm{MeV}$ [45]. For obtaining a finite-range two body contribution within the previous range, I need to vary the coordinate space cut-off $r_{c}$ between $1.1-1.4 \mathrm{fm}$.

The contribution coming from the contact range current can only be constrained by tritium beta decay. As an $\mathrm{N}^{2} \mathrm{LO}$ renormalized computation of tritium is lacking, it is impossible to estimate the size of this contribution ${ }^{5}$, meaning that our computation is incomplete at the moment.

Taking into account only those effects that can be computed in the renormalized theory presently yields the values $|\tilde{\Lambda}(0)|^{2}=7.43(2)$ and $|\tilde{\Lambda}(0)|^{2}=7.33$ (3) for set II and IV respectively. Although the previous number is of limited significance, it is bigger than the usual values obtained in most computations with phenomenological two-body currents, like $|\tilde{\Lambda}(0)|^{2}=7.06$ from Ref. [42]. It can be compared though with other chiral current computations with phenomenological wave function once the effect of the contact range operator have been removed, as $|\tilde{\Lambda}(0)|^{2}=7.49(7)$ from Ref. [43]. It is expectable though that the contribution from the contact two body operators will lower the previous results significantly. From the results of Ref. [45],

\footnotetext{
${ }^{5}$ It could be easily fixed by imposing the condition of reproducing $\Lambda(0)$, but unfortunately there is no experimental values for the $\mathrm{pp}$ fusion rate.
} 
$19^{\text {th }}$ International IUPAP Conference on Few-Body Problems in Physics

it can be seen that the contact operators give a relative negative contribution $\delta_{2 B(C)} \sim-(0.7-1.9) \%$ in the $\Lambda=$ $600-800 \mathrm{MeV}$ range, with the most negative contributions corresponding to the higher momentum space cut-offs. For the computations presented here a higher percentage is to be anticipated.

\section{Conclusions}

In this talk, the renormalization of the chiral one and two pion exchanges in the proton-proton ${ }^{1} S_{0}$ singlet channel has been considered from the point of view of boundary conditions and long range correlations. The proton-proton Coulomb phase shifts are well described up to a center of mass momentum of $k \sim 200 \mathrm{MeV}$, above which the chiral phases clearly underpredict the Nijmegen ones. This pattern of behaviour of the chiral two pion exchange phase shifts is not new [11,30,31] and persist even at $\mathrm{N}^{3} \mathrm{LO}$ [24]. Therefore it is important to determine which is missing in chiral two pion exchange to generate this persistent discrepancy in the singlet. For that, the effect of finite cut-offs and spectral regularization have been taken into account. The rather puzzling answer seems to be that the problem is the excessive strength of two pion exchanges at intermediate distances. A possible solution could be the perturbative treatment of chiral two pion exchange [20], but nonetheless it would be very interesting to see whether there are other alternative explanations from an effective field theory viewpoint.

Proton-proton fusion matrix elements can be evaluated in a straightforward manner, yielding in the impulse approximation values of the overlap integral slightly smaller than those obtained with phenomenological wave functions. This is necessary feature due to the bigger contribution of finite range axial two body currents for the renormalized computations. Unfortunately the final value of the overlap integral is still unavailable, as it would require to take into account the contact range contributions to the two body current, which so far can only be constrained by tritium beta decay. Consequently, a renormalized computation of the three nucleon bound state is a prerequisite for a complete chiral computation of proton-proton fusion.

I would like to thank T.S. Park, D.R. Phillips and E. Ruiz Arriola for discussions. This work was supported by the Helmholtz Association fund provided to the young investigator group "FewNucleon Systems in Chiral Effective Field Theory" (grant VHNG-222), the virtual institute "Spin and strong QCD" (VH-VI231), and the EU Research Infrastructure Integrating Initiative HadronPhysics2.

\section{References}

1. E. Epelbaum, Prog. Part. Nucl. Phys. 57 (2006) 654 [arXiv:nucl-th/0509032].

2. E. Epelbaum, H. W. Hammer and U. G. Meissner, arXiv:0811.1338 [nucl-th].
3. M. C. M. Rentmeester, R. G. E. Timmermans, J. L. Friar and J. J. de Swart, Phys. Rev. Lett. 82 (1999) 4992 [arXiv:nucl-th/9901054].

4. S. Weinberg, Phys. Lett. B 251 (1990) 288.

5. S. Weinberg, Nucl. Phys. B 363 (1991) 3.

6. C. Ordonez, L. Ray and U. van Kolck, Phys. Rev. Lett. 72 (1994) 1982.

7. C. Ordonez, L. Ray and U. van Kolck, Phys. Rev. C 53 (1996) 2086 [arXiv:hep-ph/9511380].

8. D. R. Entem and R. Machleidt, Phys. Rev. C 68 (2003) 041001 [arXiv:nucl-th/0304018].

9. E. Epelbaum, W. Glockle and U. G. Meissner, Nucl. Phys. A 747 (2005) 362 [arXiv:nucl-th/0405048].

10. A. Nogga, R. G. E. Timmermans and U. van Kolck, Phys. Rev. C 72, 054006 (2005) [arXiv:nuclth/0506005].

11. M. Pavon Valderrama and E. R. Arriola, Phys. Rev. C 74 (2006) 054001 [arXiv:nucl-th/0506047].

12. M. Pavon Valderrama and E. Ruiz Arriola, Phys. Rev. C 74 (2006) 064004 [Erratum-ibid. C 75 (2007) 059905] [arXiv:nucl-th/0507075].

13. E. Epelbaum and U. G. Meissner, arXiv:nuclth/0609037.

14. E. Epelbaum and J. Gegelia, Eur. Phys. J. A 41 (2009) 341 [arXiv:0906.3822 [nucl-th]].

15. M. C. Birse, Phys. Rev. C 74 (2006) 014003 [arXiv:nucl-th/0507077].

16. M. C. Birse, arXiv:0909.4641 [nucl-th].

17. M. Pavon Valderrama and E. Ruiz Arriola, Phys. Rev. C 72 (2005) 054002 [arXiv:nucl-th/0504067].

18. A. C. Cordon and E. R. Arriola arXiv:0912.1714 [cond-mat].

19. B. Long and U. van Kolck, Annals Phys. 323 (2008) 1304 [arXiv:0707.4325 [quant-ph]].

20. M. P. Valderrama, arXiv:0912.0699 [nucl-th].

21. G. P. Lepage, arXiv:nucl-th/9706029.

22. D. Shukla, D. R. Phillips and E. Mortenson, J. Phys. G 35 (2008) 115009 [arXiv:0803.4190 [nucl-th]].

23. M. Pavon Valderrama and E. R. Arriola, Annals Phys. 323, 1037 (2008) [arXiv:0705.2952 [nucl-th]].

24. D. R. Entem, E. Ruiz Arriola, M. Pavon Valderrama and R. Machleidt, Phys. Rev. C 77 (2008) 044006 [arXiv:0709.2770 [nucl-th]].

25. E. Ruiz Arriola, A. Calle Cordon and M. Pavon Valderrama, In the Proceedings of 11th International Conference on Meson-Nucleon Physics and the Structure of the Nucleon (MENU 2007), Julich, Germany, 10-14 Sep 2007, pp 161 [arXiv:0710.2770 [nucl-th]].

26. G. J. M. Austen and J. J. de Swart, Phys. Rev. Lett. 50 (1983) 2039.

27. L. Heller, Phys. Rev. 120 (1960) 627.

28. J. R. Bergervoet, P. C. van Campen, W. A. van der Sanden and J. J. de Swart, Phys. Rev. C 38 (1988) 15.

29. V. G. J. Stoks, R. A. M. Klomp, C. P. F. Terheggen and J. J. de Swart, Phys. Rev. C 49 (1994) 2950 [arXiv:nucl-th/9406039].

30. R. Higa, M. Pavon Valderrama and E. Ruiz Arriola, Phys. Rev. C 77 (2008) 034003 [arXiv:0705.4565 [nucl-th]]. 
31. M. P. Valderrama and E. R. Arriola, Phys. Rev. C 79 (2009) 044001 [arXiv:0809.3186 [nucl-th]].

32. D. R. Entem and R. Machleidt, Phys. Lett. B 524 (2002) 93 [arXiv:nucl-th/0108057].

33. E. Epelbaum, W. Gloeckle and U. G. Meissner, Eur. Phys. J. A 19 (2004) 401 [arXiv:nucl-th/0308010].

34. D. Minossi, E. Epelbaum, A. Nogga, M. Pavon Valderrama, in preparation.

35. T. Barford and M. C. Birse, Phys. Rev. C 67 (2003) 064006 [arXiv:hep-ph/0206146].

36. E. Epelbaum, W. Gloeckle and U. G. Meissner, Eur. Phys. J. A 19 (2004) 125 [arXiv:nucl-th/0304037].

37. C. J. Yang, C. Elster and D. R. Phillips, arXiv:0909.5414 [nucl-th].

38. C. J. Yang, C. Elster and D. R. Phillips, Phys. Rev. C 80 (2009) 044002 [arXiv:0905.4943 [nucl-th]].

39. C. J. Yang, C. Elster and D. R. Phillips, Phys. Rev. C 80 (2009) 034002 [arXiv:0901.2663 [nucl-th]].

40. J. Carlson, D. O. Riska, R. Schiavilla and R. B. Wiringa, Phys. Rev. C 44 (1991) 619.

41. M. C. M. Rentmeester, R. G. E. Timmermans and J. J. de Swart, Phys. Rev. C 67 (2003) 044001 [arXiv:nucl-th/0302080].

42. R. Schiavilla et al., Phys. Rev. C 58 (1998) 1263 [arXiv:nucl-th/9808010].

43. T. S. Park, K. Kubodera, D. P. Min and M. Rho, Astrophys. J. 507 (1998) 443 [arXiv:astro-ph/9804144].

44. T. S. Park, D. P. Min and M. Rho, Phys. Rept. 233 (1993) 341 [arXiv:hep-ph/9301295].

45. T. S. Park et al., Phys. Rev. C 67 (2003) 055206 [arXiv:nucl-th/0208055]. 